\title{
Value of Additional Digital Breast Tomosynthesis for Preoperative Staging of Breast Cancer in Dense Breasts
}

\author{
JULIA KRAMMER ${ }^{1}$, KATHRIN STEPNIEWSKI ${ }^{1}$, CLEMENS G. KAISER $^{1}$, JOACHIM BRADE $^{2}$, \\ PHILIPP RIFFEL ${ }^{1}$, STEFAN O. SCHOENBERG ${ }^{1}$ and KLAUS WASSER ${ }^{1}$ \\ ${ }^{1}$ Institute of Clinical Radiology and Nuclear Medicine, University Medical Center Mannheim, and \\ ${ }^{2}$ Institute of Medical Statistics, Biomathematics and Data Processing, \\ Medical Faculty Mannheim, University of Heidelberg, Mannheim, Germany
}

\begin{abstract}
Aim: This retrospective study was initiated to determine the diagnostic value of additional preoperative breast tomosynthesis (DBT) for breast cancer staging in dense breasts. Patients and Methods: Sixty-six patients (69 breasts) with findings of American College of Radiology category 3 or 4 with Breast Imaging Reporting and Data System 5, 6 or 0 were included. All patients underwent digital mammography and additional DBT. Results: A total of 40/69 (58\%) cancers were detected on both mammography and DBT, 23 (33.3\%) were only seen on DBT ( $p=0.0001)$; $6 / 69$ (8.7\%) carcinomas were not detected by either method, of which three were invasive lobular carcinomas. Sensitivity for multifocal/multicentric disease was significantly higher on DBT $(12 / 19,63.2 \%)$ compared to mammography $(4 / 19,21.1 \%)(p=0.02)$, specificity was comparable ( $96.0 \%$ vs. $90.0 \%)$. Multifocal/multicentric disease was not detected on mammography nor DBT in 7/19 (36.8\%) patients, including three invasive lobular carcinomas. Conclusion: DBT may significantly improve preoperative breast cancer staging in patients with dense breasts compared to conventional mammography alone. Nevertheless, limitations have to be expected in the case of invasive lobular carcinoma.

Breast cancer tumor stage at initial diagnosis determines prognosis and directs treatment planning. With the increasing use of neoadjuvant chemotherapy and breast-conserving therapy, a precise definition of the tumor size and tumor number is more essential than ever for patient selection. According to
\end{abstract}

Correspondence to: Julia Krammer, Institute of Clinical Radiology and Nuclear Medicine, University Medical Centre Mannheim, Medical Faculty Mannheim, University of Heidelberg, TheodorKutzer-Ufer 1-3, 68167 Mannheim, Germany. Tel: +49 06213832067, Fax: +49 06213833817, e-mail: Julia.Krammer@ medma.uni-heidelberg.de

Key Words: Breast tomosynthesis, staging, breast cancer, mammography. the national guidelines, the disease extent is usually determined by means of clinical examination, conventional mammography and breast ultrasound; additional breast magnetic resonance imaging (MRI) may be performed in selected cases $(1,2)$. However, sensitivity of mammography can be limited in areas of high breast density as overlying tissue can obscure tumors and hinder correct estimation of tumor size.

Digital breast tomosynthesis (DBT) has emerged as a promising new imaging technique, which seems to overcome the limitations of mammography by reducing the obscuring effect of overlying breast tissue. Large prospective studies have described that integrated DBT may improve breast cancer detection and reduce recall rates in screening populations (3-5). Moreover, studies evaluating patients with known breast lesions showed promising results regarding the evaluation of the tumor size and visibility using DBT (6-9). Despite intensified research on the value of DBT for lesion characterization and size determination, studies evaluating its ability to determine disease focality are still lacking. This information is essential as it has an impact on the patient's surgical management and treatment planning. Furthermore, only few data exist about the applicability and diagnostic value of DBT in patients with high breast density according to American College of Radiology (ACR) category 3 and ACR $4(10,11)$. This retrospective study was designed to address these issues: assessing the value of integrating DBT in the preoperative local staging of breast cancer with focus on the detection of multifocal/multicentric disease in a patient population with dense breasts on conventional mammography.

\section{Patients and Methods}

Patient population. This retrospective study was approved by the National Federal Radiation Commission and the Institutional Review Board (2014-803R-MA). Data analysis was performed in accordance with the Health Insurance Portability and Accountability Act and the Declaration of Helsinki.

The study was conducted between 01/2010 and 03/2014. DBT was performed in addition to conventional mammography if patients 
met the following criteria: mammographic dense breast parenchyma of ACR 3 or 4 and Breast Imaging Reporting and Data System (BIRADS) 5 or 6 findings on diagnostic conventional digital mammography or BI-RADS 0 with suspicious clinical findings. DBT was not routinely performed bilaterally, only in cases where both breasts fulfilled the inclusion criteria. Sixty-six patients (69 breasts) were included and findings were evaluated retrospectively: 6/66 $(9.1 \%)$ patients underwent mammography for routine screening, 5/66 (7.6\%) for aftercare following contralateral breast cancer. Overall, $55 / 66(83.3 \%)$ patients presented with suspicious clinical symptoms and mammography was obtained for further diagnostic workup. Findings in 38/69 (55.1\%) breasts were classified as BI-RADS 5 and $10 / 69$ (14.5) as BI-RADS 6. Conventional mammography was classified as BI-RADS 0 in 21/69 (30.4\%) cases with the following clinical findings: 17 cases with a new palpable mass, one case with nipple retraction, three cases with suspicious ultrasound findings.

Sixty-three out of 66 patients $(95.5 \%)$ underwent unilateral DBT. Bilateral DBT was only performed in the case of unclear findings on unilateral DBT. In this study, $3 / 66$ patients $(4.5 \%)$ underwent bilateral DBT.

Reference standard. Histopathology served as reference standard in 44/69 (63.8\%) breasts. In 16/44 (36.4\%), additional pre-operative breast MRI was available to reinforce histopathology. If patients received neoadjuvant chemotherapy or did not undergo final surgery due to a palliative approach, a combination of ultrasound, breast MRI and biopsy of the lesions, as well as follow up imaging of at least 2 years, served as reference standard $(n=25,36.2 \%)$.

Image analysis. Conventional mammography and DBT were compared by two radiologists in consensus, one with at least 15 years' experience in breast imaging including 4 years of DBT reading and one with 4 years of experience in breast imaging including 2 years of DBT reading. Conventional mammography was read first without knowledge of the DBT images. Afterwards, DBT images were considered. The visibility of lesions, lesion characteristics, disease focality, as well as the size and BI-RADS classification of each lesion were evaluated. A change of size measurement on DBT compared to conventional mammography was considered as significant if it led to a change of the T stage. Furthermore, a change of size of $10 \mathrm{~mm}$ or more was considered as significant, since this led to an adaption of the surgical procedure with a wider resection in a breast-conserving approach. All findings were correlated to the reference standard and the diagnostic value of mammography alone versus mammography in combination with DBT was finally determined.

Image acquisition. Two plain (cranio-caudal and medio-lateraloblique) conventional mammographic images as well as DBT images were acquired using a Mammomat Inspiration Siemens (Siemens Healthcare Sector, Erlangen, Germany). The mean absorbed dose for a one plain conventional mammography of a standard breast was $1.6 \mathrm{mGy}$ and $1.1 \mathrm{mGy}$ for DBT. Fifteen projection images were acquired over an angular range of approximately $50^{\circ}\left( \pm 25^{\circ}\right.$ around the mediolateral oblique/ craniocaudal position). A 3D dataset with $1 \mathrm{~mm}$ slice thickness was acquired using filtered back projection.

Statistical analysis. Frequencies and percentages were used to summarize categorical variables. Medians, ranges and means were used to summarize continuous variables. Sensitivity and specificity were calculated. Chi-square test was used to demonstrate statistical significance. A $p$-value of less than 0.05 was considered to be statistically significant. All statistical analyses were performed using SPSS 13.0 statistical package (SPSS Inc., Chicago, IL, USA).

\section{Results}

A total of 69 breasts in 66 patients (median age $=61.5$ years, range $=36-81$ years) were evaluated. Invasive cancer was found in all breasts. The median histological tumor size was $20.5 \mathrm{~mm}$ (range=2-86 $\mathrm{mm}$ ).

Primary lesion. Overall, 40/69 (58\%) primary lesions were detected on both conventional mammography and DBT, whereas 23/69 (33.3\%) tumors were only seen on DBT ( $p \leq 0.0001)$. The overall detection rate on conventional mammography was $58.0 \%$ compared to $91.3 \%$ on DBT. Six primary lesions were not detected on conventional mammo-graphy nor DBT; three of these were invasive lobular carcinomas and three were carcinomas of no specific type. One of the false-negative invasive lobular carcinomas is displayed in Figure 1. The detection rates by mammography and DBT according to the histology of the primary lesion are displayed in Table I.

Multifocal or multicentric disease. Multifocal or multicentric disease was present in 19/69 (27.5\%) breasts. Conventional mammography detected multifocality or multicentricity in 4/19 breasts and DBT in 12/19 breasts, with a corresponding sensitivity of $21.1 \%$ and $63.2 \%$ $(p=0.02)$ (Figure 2). The corresponding specificities did not significantly differ at $96.0 \%$ and $90.0 \%(p>0.05)$ (Table II). In seven patients, multifocal or multicentric disease was not detected on either conventional mammography or DBT. Three of these patients had an invasive lobular carcinoma, three a carcinoma of no specific type, and one had a mucinous carcinoma.

DBT was falsely positive for additional disease in 5/69 (7.2\%) breasts; two of these $(2.9 \%)$ had a correlating falsepositive finding on mammography $(p=0.44)$. The false-positive lesions were found to be a fibroadenoma, a fatty necrosis, a calcified cyst and normal breast parenchyma. Three of the breasts had unifocal invasive lobular carcinomas and two a unifocal tumor of no specific type as primary disease.

Lesion size. Overall 97 lesions, 69 primary lesions and 28 satellite lesions, were registered in 69 breasts. Of these, 44 (45.4\%) were detectable on both mammography and DBT. The median tumor size of these lesions was $29 \mathrm{~mm}$ (range $=7-75 \mathrm{~mm}$ ) on conventional mammography and 26 $\mathrm{mm}$ (range $=8-88 \mathrm{~mm}$ ) on DBT.

The size measurement on mammography and DBT did not significantly differ in 34/44 $(77.3 \%)$ lesions and was concordant with the reference standard in 34/44 breasts $(77.3 \%)$. In six patients, mammography and DBT 


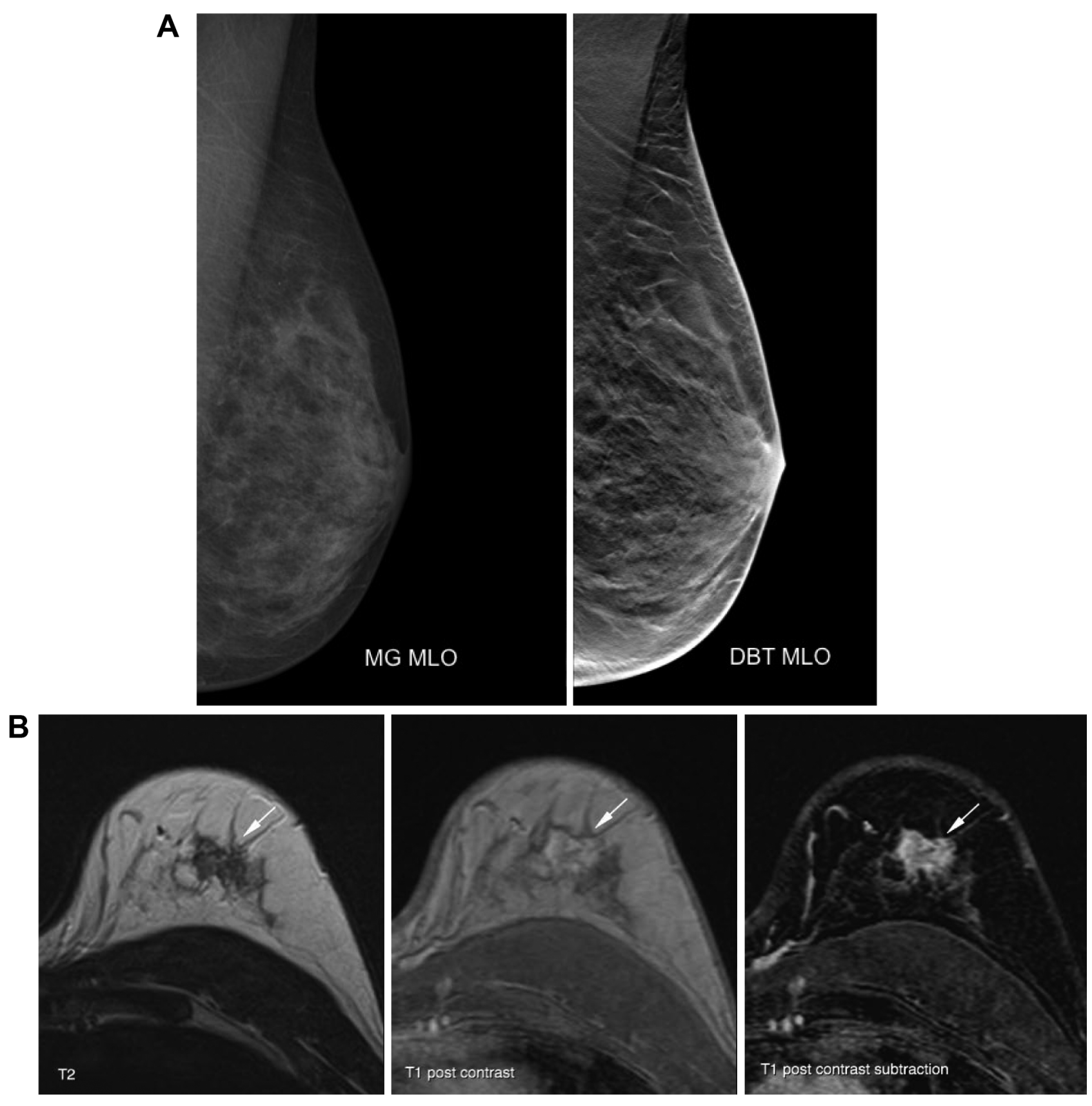

Figure 1. A 75-year-old patient with dense breasts (American College of Radiology category 3) and a palpable tumor in her left upper quadrant. A: Mammography $(M G)$ and digital breast tomosynthesis (DBT) showed no suspicious finding (craniocaudal view not shown). B: Breast magnetic resonance imaging revealed an $18 \mathrm{~mm}$ spiculated mass-like enhancement consistent with invasive lobular cancer, which was occult on MG and DBT (white arrows). MLO: Mediolateral oblique view.

overestimated the tumor size, in four patients, the tumor was underestimated. In comparison to conventional mammography, DBT led to a significant change of size measurement in 10/44 $(22.7 \%)$ lesions: an increase in size in six cases and a decrease in size in four cases (Figure 3).

\section{Discussion}

In this study we showed that additional DBT may significantly improve preoperative local staging of breast cancer in patients with dense breasts. 


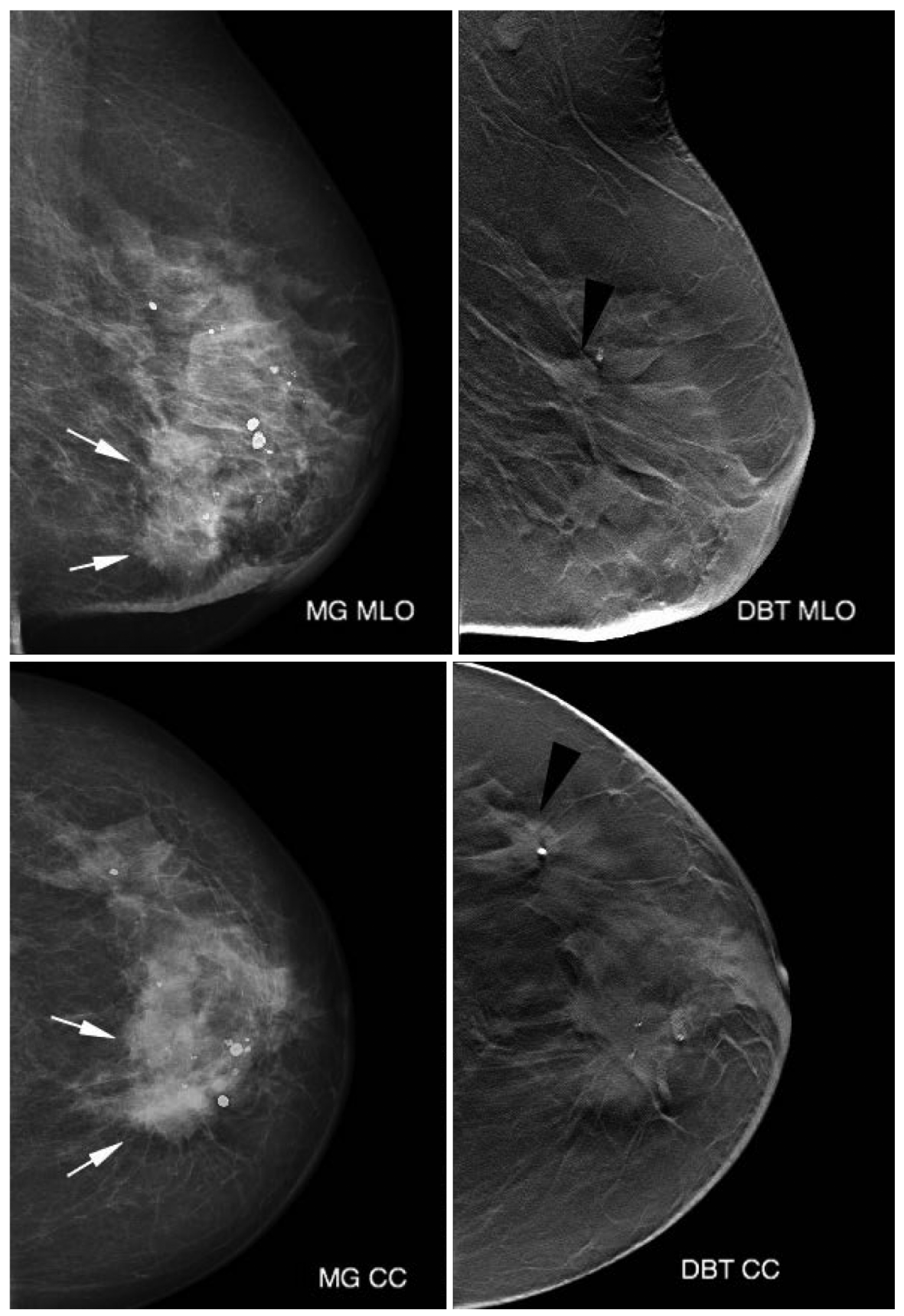

Figure 2. Mammography $(M G)$ and digital breast tomosynthesis (DBT) of a 77-year-old patient with a 3.7 cm invasive carcinoma of no specific type in the middle of her left breast (white arrows). DBT showed two additional tumor sites in the outer quadrant and prepectoral region (black arrows) not seen on MG. Based on DBT, tumor stage was changed from unifocal to multicentric disease. CC: Craniocaudal view; MLO: mediolateral oblique view.

DBT was superior to mammography in the detection of the primary lesion, with a significantly higher detection rate of $91.3 \%$ compared to $58 \%(p \leq 0.0001)$. Comparing this to literature Andersson et al. included 40 patients with cancer with varying breast density and found a better visibility of the primary tumor on DBT in $27.5 \%$ (7). Mariscotti et al. conducted a large study of 200 breast cancer patients and found a slightly improved sensitivity of DBT compared to mammography with $91 \%$ vs. $85 \%$ (12). However, both studies included patients with varying breast density, which 
Table I. Histology-based detection rates of primary lesions on mammography (MG) and digital breast tomosynthesis (DBT) ( $n=69)$.

\begin{tabular}{|c|c|c|c|c|}
\hline \multirow[b]{2}{*}{ Histological subtype } & \multicolumn{2}{|c|}{ Detection rate, $\mathrm{n}(\%)$} & \multirow[t]{2}{*}{ Total $(\%)$} & \multirow[t]{2}{*}{$p$-Value } \\
\hline & MG & DBT $(\%)$ & & \\
\hline Total & $40(58.0 \%)$ & $63(91.3)$ & $69(100)$ & $<0.0001$ \\
\hline NST carcinoma & $29(42.0)$ & $40(58.0)$ & $43(62.3)$ & \\
\hline Invasive lobular carcinoma & $7(10.1)$ & $15(21.7)$ & $18(26.1)$ & \\
\hline Mixed invasive ductal-lobular carcinoma & $1(1.4)$ & $2(2.9)$ & $2(2.9)$ & \\
\hline Other & $3(4.3)$ & $6(8.7)$ & $6(8.7)$ & \\
\hline
\end{tabular}

NST: No specific type.

Table II. Detection of multifocal or multicentric disease on mammography (MG) and digital breast tomosynthesis DBT ( $n=19)$.

\begin{tabular}{lccc}
\hline & MG & DBT & $p$-Value \\
\hline Correctly identified, $\mathrm{n} / \mathrm{N}$ & $4 / 19$ & $12 / 19$ & 0.02 \\
Sensitivity (95\% CI) & $21.1 \%(2.8-39.4 \%)$ & $63.2 \%(41.5-84.9 \%$ & $>0.05$ \\
Specificity (95\% CI) & $96.0 \%(90.6-101.4 \%)$ & $90.0 \%(81.7-98.3 \%)$ & $70.6 \%$ \\
Positive predictive value & $66.7 \%$ & $86.5 \%$ & \\
Negative predictive value & $76.1 \%$ & & \\
\hline
\end{tabular}

CI: Confidence interval.

might explain the lower differences in the overall sensitivities compared to our study, which only included patients with dense breasts. Assessing only patients with dense breast parenchyma, sensitivity significantly decreased for mammography but not for DBT in their studies. With nearly the same detection rate of $91.3 \%$ for DBT, our results are in line with these findings.

Secondly, we found a positive impact of additional DBT on the detection rate of multifocal or multicentric disease. Bian et al. showed higher overall detection rates using DBT compared to mammography in a study population of 631 breasts assessing benign as well as malignant lesions (13). However, to our knowledge, no data exist on the value of additional DBT in a population of patients with dense breasts. Regarding the detection of additional disease in this challenging population, to our knowledge, this is the first study showing that adding DBT to a conventional mammography substantially increases sensitivity for multifocal/multicentric disease from $21.1 \%$ to $63.2 \%(p=0.02)$. However, despite a superior performance of DBT compared to mammography, it is still not as good as breast MRI in the detection of additional tumor sites, with described sensitivities of up to $100 \%$ (14).

Regarding the size measurement, 10 lesions (22.7\%) were correctly changed in size based on DBT. This trend was also described by other studies. Seo et al. found a stronger correlation of the size evaluation obtained by DBT compared to mammography regardless of the parenchymal density in
114 women with a single breast lesion (10). Mun et al. described a decrease of mis-sizing in $10 \%$ using DBT compared to mammography; in their study of 169 patients, two-thirds had heterogeneously or extremely dense breasts (11). Luparia et al. retrospectively reviewed 146 breast cancers and found a $12 \%$ higher level of concordance with pathology using DBT (66\%) compared to mammography (54\%) (8).

Interestingly, regarding the breasts with false-negative DBT (i.e. six primary lesions and seven breasts with additional tumor sites), three of these had an invasive lobular carcinoma. Consequently, limited accuracy of DBT for the local staging of invasive lobular carcinoma should be expected. This limitation is described for mammography as well, with false-negative rates of up to $20 \%(15,16)$. This is mainly due to the specific histological features of this tumor type. A lack of stromal desmoplasia and an infiltrative growth pattern more often leads to subtle findings with focal asymmetry and architectural distortions, which are harder to detect on mammography. Nevertheless, in this study, additional DBT detected more invasive lobular carcinomas in dense breasts than mammography alone, with an increase of sensitivity from $38.9 \%(7 / 18)$ to $83.3 \%(15 / 18)$. Mariscotti et al. recently confirmed in a retrospective analysis of 83 patients that combining mammography with DBT significantly increases the detection of invasive lobular carcinoma (17). However, they included patients with varying breast densities, so comparability is limited. 


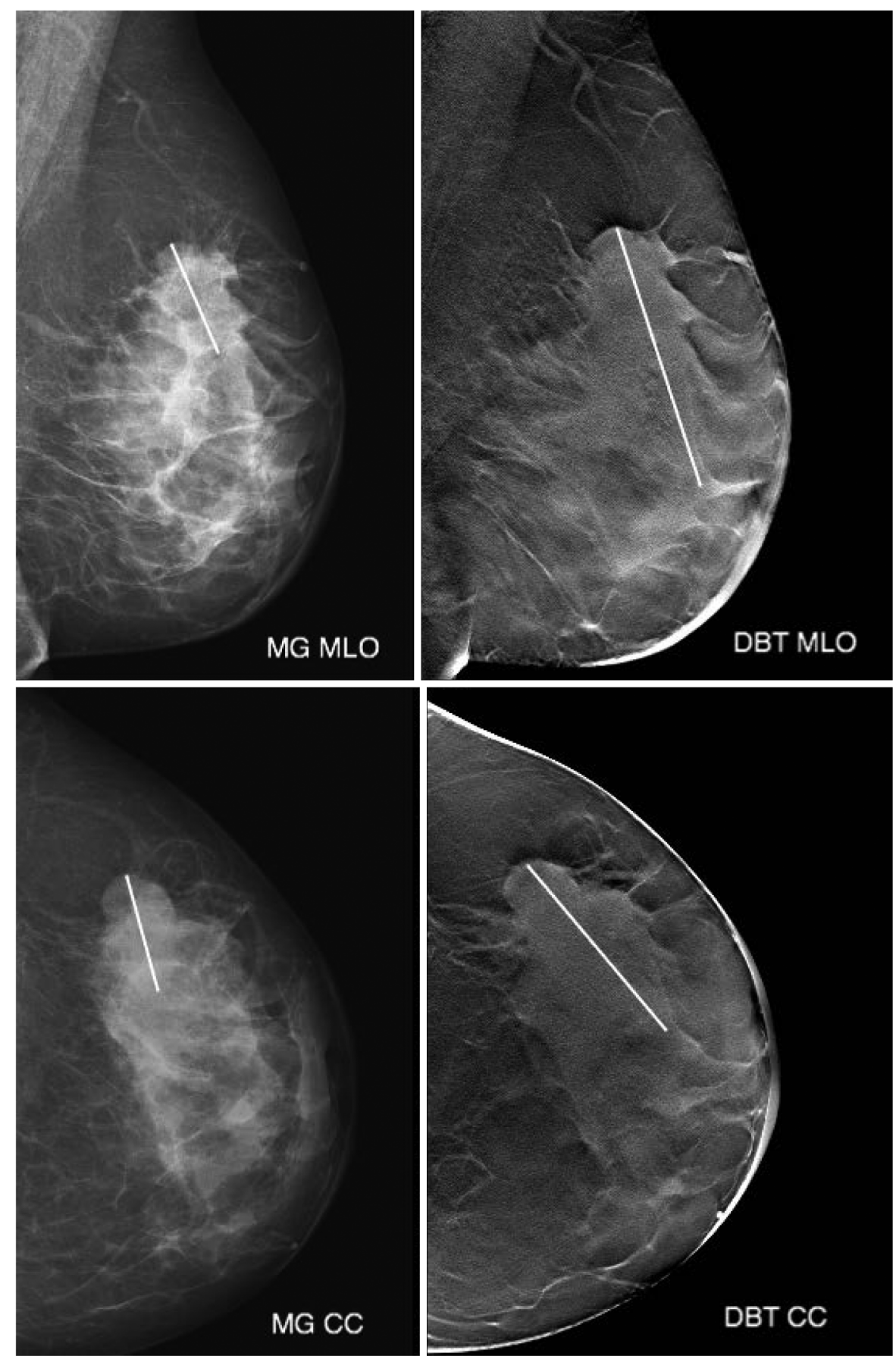

Figure 3. Conventional digital mammography $(M G)$ and digital breast tomosynthesis (DBT) showing mediolateral oblique (MLO) and craniocaudal (CC) views of a 60-year-old woman with a suspicious palpation finding in her left upper outer quadrant. The corresponding tumor was initially seen on mammography with a size of $24 \times 26 \mathrm{~mm}$. Size measurement on DBT was significantly larger at $48 \times 60 \mathrm{~mm}$ (compare white bars).

Our study has several limitations. Firstly, this was a singlecenter study with a retrospective study design. Furthermore, in cases of a palliative approach or neoadjuvant therapy $(\mathrm{n}=25)$, the final pathology was not available or not suitable for comparison. In these cases, the combination of biopsy, preoperative breast MRI and ultrasound served as reference standard. Multiple studies are concordant that breast MRI is the most accurate imaging method to map the extent of local 
disease and to identify additional tumor sites, if interpreted when corresponding mammography, ultrasound and biopsy is taken into account. Consequently, we regard this diagnostic combination as sufficient counterpart in these patients $(18,19)$.

Regarding image analysis, a potential bias in the detection rates might arise because images were interpreted by readers with knowledge of the prior mammography. However, we do not believe this impacts our conclusion. The design of this study specified DBT as an additional imaging tool for staging purposes and did not aim to replace initial digital mammography by DBT alone. Rather it reflects its use in clinical routine.

In conclusion, DBT in addition to mammography can significantly improve preoperative local staging of breast cancer, especially in patients with dense breasts. DBT is superior to mammography in the detection of the primary lesion and detects significantly more additional tumor sites in dense breasts. This may aid in determining optimal treatment in these patients, i.e. a more appropriate selection of patients better served with mastectomy rather than a breast-conserving therapy approach. Applying DBT in addition to mammography as part of the preoperative work-up of breast cancer in patients with dense breasts may be a cost- and timeeffective alternative, especially if preoperative breast MRI is not available or patients have contraindications for MRI. However, the sensitivity cannot exceed that of breast MRI and limitations have to be expected in the case of invasive lobular carcinoma. Larger prospective studies are needed to confirm our results and to pave the way for a standard use of DBT for preoperative workup in patients with dense breasts.

\section{References}

1 Kreienberg R: Interdisciplinary S3 Guidelines for the Diagnosis, Treatment and Follow-up Care Breast Cancer, 3rd updated version. München: Zuckerschwerdt Verlag, 2012.

2 Senkus E, Kyriakides S, Penault-Llorca F, Poortmans P, Thompson A, Zackrisson S, Cardoso F and Group EGW: Primary breast cancer: ESMO Clinical Practice Guidelines for diagnosis, treatment and follow-up. Ann Oncol 24(Suppl 6): vi7-23, 2013

3 Rafferty EA, Park JM, Philpotts LE, Poplack SP, Sumkin JH, Halpern EF and Niklason LT: Assessing radiologist performance using combined digital mammography and breast tomosynthesis compared with digital mammography alone: results of a multicenter, multireader trial. Radiology 266: 104-113, 2013.

4 Ciatto S, Houssami N, Bernardi D, Caumo F, Pellegrini M, Brunelli S, Tuttobene P, Bricolo P, Fanto C, Valentini M, Montemezzi S and Macaskill P: Integration of 3D digital mammography with tomosynthesis for population breast-cancer screening (STORM): a prospective comparison study. Lancet Oncol 14: 583-589, 2013.

5 Skaane P, Bandos AI, Gullien R, Eben EB, Ekseth U, Haakenaasen U, Izadi M, Jebsen IN, Jahr G, Krager M, Niklason LT, Hofvind S and Gur D: Comparison of digital mammography alone and digital mammography plus tomosynthesis in a population-based screening program. Radiology 267: 47-56, 2013.

6 Poplack SP, Tosteson TD, Kogel CA and Nagy HM: Digital breast tomosynthesis: initial experience in 98 women with abnormal digital screening mammography. AJR Am J Roentgenol 189: 616-623, 2007.

7 Andersson I, Ikeda DM, Zackrisson S, Ruschin M, Svahn T, Timberg P and Tingberg A: Breast tomosynthesis and digital mammography: a comparison of breast cancer visibility and BIRADS classification in a population of cancers with subtle mammographic findings. Eur Radiol 18: 2817-2825, 2008.

8 Luparia A, Mariscotti G, Durando M, Ciatto S, Bosco D, Campanino PP, Castellano I, Sapino A and Gandini G: Accuracy of tumour size assessment in the preoperative staging of breast cancer: comparison of digital mammography, tomosynthesis, ultrasound and MRI. La Radiologia medica 118: 1119-1136, 2013.

9 Fornvik D, Zackrisson S, Ljungberg O, Svahn T, Timberg P, Tingberg A and Andersson I: Breast tomosynthesis: Accuracy of tumor measurement compared with digital mammography and ultrasonography. Acta Radiol 51: 240-247, 2010.

10 Seo N, Kim HH, Shin HJ, Cha JH, Kim H, Moon JH, Gong G, Ahn SH and Son BH: Digital breast tomosynthesis versus fullfield digital mammography: comparison of the accuracy of lesion measurement and characterization using specimens. Acta Radiol 55: 661-667, 2014.

11 Mun HS, Kim HH, Shin HJ, Cha JH, Ruppel PL, Oh HY and Chae EY: Assessment of extent of breast cancer: comparison between digital breast tomosynthesis and full-field digital mammography. Clin Radiol 68: 1254-1259, 2013.

12 Mariscotti G, Houssami N, Durando M, Bergamasco L, Campanino PP, Ruggieri C, Regini E, Luparia A, Bussone R, Sapino A, Fonio $\mathrm{P}$ and Gandini G: Accuracy of mammography, digital breast tomosynthesis, ultrasound and MR imaging in preoperative assessment of breast cancer. Anticancer Res 34: 1219-1225, 2014

13 Bian T, Lin Q, Cui C, Li L, Qi C, Fei J and Su X: Digital breast tomosynthesis: A new diagnostic method for mass-like lesions in dense breasts. Breast J 22: 535-540, 2016.

14 Houssami N, Ciatto S, Macaskill P, Lord SJ, Warren RM, Dixon $\mathrm{JM}$ and Irwig L: Accuracy and surgical impact of magnetic resonance imaging in breast cancer staging: systematic review and meta-analysis in detection of multifocal and multicentric cancer. J Clin Oncol 26: 3248-3258, 2008.

15 Veltman J, Boetes C, van Die L, Bult P, Blickman JG and Barentsz JO: Mammographic detection and staging of invasive lobular carcinoma. Clin Imaging 30: 94-98, 2006.

16 Hilleren DJ andersson IT, Lindholm K and Linnell FS: Invasive lobular carcinoma: mammographic findings in a 10-year experience. Radiology 178: 149-154, 1991.

17 Mariscotti G, Durando M, Houssami N, Zuiani C, Martincich L, Londero V, Caramia E, Clauser P, Campanino PP, Regini E, Luparia A, Castellano I, Bergamasco L, Sapino A, Fonio P, Bazzocchi M and Gandini G: Digital breast tomosynthesis as an adjunct to digital mammography for detecting and characterising invasive lobular cancers: a multi-reader study. Clin Radiol 71: 889-895, 2016.

18 Sardanelli F: Overview of the role of pre-operative breast MRI in the absence of evidence on patient outcomes. Breast 19: 3-6, 2010.

19 Kuhl C, Kuhn W, Braun M and Schild H: Pre-operative staging of breast cancer with breast MRI: one step forward, two steps back? Breast 16(Suppl 2): S34-44, 2007.

Received July 11, 2017

Revised July 29, 2017

Accepted August 2, 2017 